\title{
Mast cells play a key role in Th2 cytokine-dependent asthma model through production of adhesion molecules by liberation of TNF- $\alpha$
}

\author{
Ok Hee Chai ${ }^{1 *}$, Eui-Hyeog Han ${ }^{1 *}$, \\ Hern-Ku Lee ${ }^{2}$ and Chang Ho Song ${ }^{1,3}$ \\ ${ }^{1}$ Department of Anatomy \\ Chonbuk National University Medical School \\ and Institute for Medical Sciences \\ Chonbuk National University \\ Jeonju 561-756, Korea \\ 2Department of Immunology \\ Chonbuk National University Medical School \\ Jeonju 561-756, Korea \\ ${ }^{3}$ Corresponding author: Tel, 82-63-270-3101; \\ Fax, 82-63-274-9880; E-mail, asch@jbnu.ac.kr \\ *These authors contributed equally to this work. \\ DOI 10.3858/emm.2011.43.1.004
}

Accepted 17 December 2010

Available Online 20 December 2010

Abbreviations: $\mathrm{BAL}$, bronchoalveolar lavage; $\mathrm{BMCMC} \rightarrow W / W^{N}$, mast cell-reconstituted $W / W^{W}$; BMCMCs, bone marrow-derived cultured mast cells; $\mathrm{H}-\mathrm{E}$, hematoxylin and eosin; ICAM, intercellular adhesion molecule; OVA, ovalbumin; PAS, periodic acid-Schiff; PenH, enhanced respiratory pause; Th2, T-helper type 2; VCAM, vascular cellular adhesion molecule; $W / W^{N}$, mast cell-deficient WBB6F1/ $\mathrm{J}-\mathrm{Kit}^{W} / \mathrm{Kit}^{\mathrm{W}-\mathrm{v}} ;+/+$, congenic normal WBB6F1/J-Kit $/ \mathrm{Kit}^{+}$

\begin{abstract}
Mast cells are well recognized as key cells in allergic reactions, such as asthma and allergic airway diseases. However, the effects of mast cells and TNF- $\alpha$ on T-helper type 2 (Th2) cytokine-dependent asthma are not clearly understood. Therefore, an aim of this study was to investigate the role of mast cells on Th2 cytokine-dependent airway hyperresponsiveness and inflammation. We used genetically mast cell-deficient WBB6F1/J-Kit ${ }^{W} \mid K i t^{W-v}(W / W)$, congenic normal WBB6F1/J-Kit $\mid \mathrm{Kit}^{+}(+/+)$, and mast cell-reconstituted $W / W^{W}$ mouse models of allergic asthma to investigate the role of mast cells in Th2 cytokine-dependent asthma induced by ovalbumin (OVA). And we investigated whether the intratracheal injection of TNF- $\alpha$ directly induce the expression of ICAM-1 and VCAM-1 in W/W mice. This study, with OVA-sensitized and OVA-challenged mice, revealed the following typical histopatho-
\end{abstract}

logic features of allergic diseases: increased inflammatory cells of the airway, airway hyperresponsiveness, and increased levels of TNF- $\alpha$, intercellular adhesion molecule (ICAM)-1, and vascular cellular adhesion molecule (VCAM)-1. However, the histopathologic features and levels of ICAM-1 and VCAM-1 proteins in $W / W^{\prime}$ mice after OVA challenges were significantly inhibited. Moreover, mast cell-reconstituted $W / W^{N}$ mice showed restoration of histopathologic features and recovery of ICAM-1 and VCAM-1 protein levels that were similar to those found in +/+ mice. Intratracheal administration of TNF- $\alpha$ resulted in increased ICAM-1 and VCAM-1 protein levels in $W / W^{N}$ mice. These results suggest that mast cells play a key role in a Th2 cytokine-dependent asthma model through production of adhesion molecules, including ICAM- 1 and VCAM-1, by liberation of TNF- $\alpha$.

Keywords: asthma; cell adhesion molecules; mast cells; models, animal; ovalbumin; Th2 cells; tumor necrosis factor- $\alpha$

\section{Introduction}

Allergic asthma is a chronic inflammatory disorder of the airways, which is characterized by variable airway obstruction, airway eosinophilic inflammation, and airway hyperresponsiveness (Cohn et al., 2004). Airway inflammation in atopic asthma is associated with a T-helper type 2 (Th2) immune response in which Th2 cell-derived cytokines are thought to contribute to eosinophil recruitment, mucus hypersecretion, and airway hyperresponsiveness (Cohn et al., 2004; Woodruff et al., 2009; Kim et al., 2010).

Mast cells are the main effector cells of especially the early phase of the allergic reaction and immediated asthmatic response. Through antigen cross-linking to high affinity $\lg E$ receptors on the cell surface, mast cells discharge a group of mediators (Galli and Tsai, 2008), including histamine. Mast cell-derived lipid mediators and inflammatory cytokines, such as leukotrienes, IL-6, and TNF- $\alpha$, have also been reported to play an important role in the late asthmatic response and airway hyperresponsiveness (Matsuoka et al., 2000; 


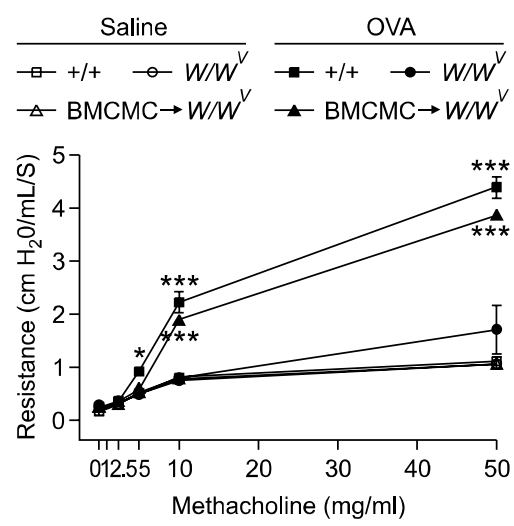

Figure 1. Resistance of mice to methacholine after saline sensitization and challenge or ovalbumin (OVA) sensitization and challenge in $+/+$, $W W^{N}$, and $\mathrm{BMCMC} \rightarrow W / W^{N}$ mice. Data represent the mean \pm SEM of 5 mice in each group. ${ }^{*} P<0.05,{ }^{* \star *} P<0.001$ versus $W / W^{\prime}$ (OVA).

Zimmermann et al., 2003; Antunez et al., 2006; Woodruff et al., 2009). TNF- $\alpha$ is a chemotactic cytokine for granulocytes, including eosinophils and neutrophils (Sabatini et al., 2002; Chai et al., 2005; Yoshifuku et al., 2007), probably by upregulation of cellular adhesion molecules, such as vascular cellular adhesion molecule (VCAM)-1, and intercellular adhesion molecule (ICAM)-1, thus facilitating migration of eosinophils and neutrophils. During this process, they also become primed for mediator secretion. Ultimately, this will lead to chronic inflammation and irreversible airway remodeling, a key feature in bronchial asthma. However the role of TNF- $\alpha$ in allergic inflammation is still controversial. Also, the relationship between TNF- $\alpha$ and adhesion molecules in asthma is not clear.

Therefore, in this study, we investigated the possible roles of mast cells with regard to certain parameters, such as ICAM-1 and VCAM-1, of allergic inflammation in a Th2 cytokine-dependent asthma model using genetically mast cell-deficient WBB6F1/J-Kit ${ }^{W} / K i t^{W-v}\left(W / W^{v}\right)$, congenic normal WBB6F1/J-Kit $/ \mathrm{Kit}^{+}(+/+)$, and mast cell-reconstituted $W / W^{v}\left(B M C M C \rightarrow W / W^{v}\right)$ mice. And we investigated whether the intratracheal injection of TNF- $\alpha$ directly induce the expression of ICAM-1 and VCAM-1 in W/W mice. We found that mast cells are essential in development of Th2 cytokine-dependent asthma, because of the liberation of TNF- $\alpha$, which activates and enhances production of ICAM-1 and VCAM-1.

\section{Results}

\section{Airway responsiveness to methacholine in mice after OVA challenge}

The dose curve of PenH responses to aerosolized methacholine of OVA-sensitized and -challenged mice shifted to the left, compared with that of saline-sensitized and -challenged mice (Figure 1). PenH responses to aerosolized methacholine increased significantly in OVA-sensitized and -challenged $+/+$ mice and $\mathrm{BMCMC} \rightarrow W / W^{\nu}$ mice, compared with those of saline-sensitized and -challenged $+/+W / W^{\nu}$, and $\mathrm{BMCMC} \rightarrow W / W^{\nu}$ mice. PenH responses in OVA-sensitized and -challenged $W / W^{V}$ mice were significantly lower than those in OVA-sensitized and -challenged $+/+$ mice at $50 \mathrm{mg} / \mathrm{ml}$ of methacholine inhalation. However, PenH responses in OVA-sensitized and -challenged $\mathrm{BMCMC} \rightarrow W / W^{\nu}$ mice were significantly higher at $50 \mathrm{mg} / \mathrm{ml}$ of methacholine inhalation than in OVA-sensitized and -challenged $W / W^{V}$ mice. PenH responses to aerosolized methacholine in BMCMC

A
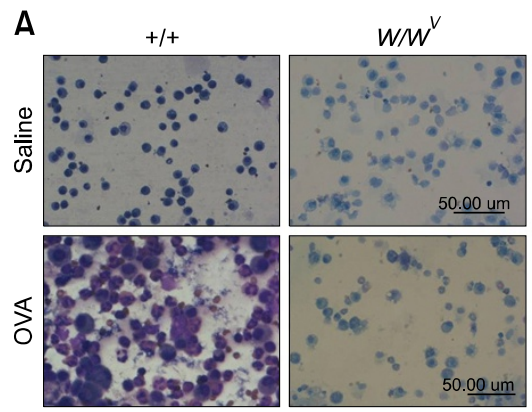

$\mathrm{BMCMC} \rightarrow W W^{V}$

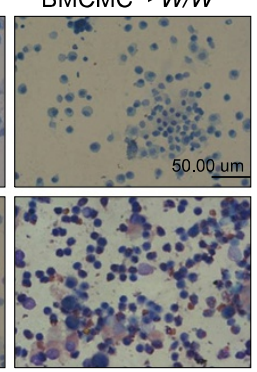

B

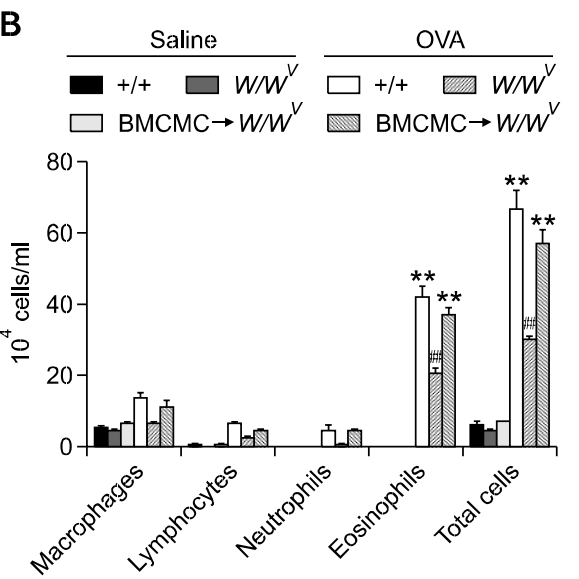

Figure 2. Infiltration of (A) inflammatory cells and (B) differential cellular components and total cells in bronchoalveolar lavage fluid after saline sensitization and challenge (Saline) or ovalbumin sensitization and challenge $(\mathrm{OVA})$ in $+/+, W / W^{N}$, and $\mathrm{BMCMC} \rightarrow W / W^{N}$ mice. ${ }^{* *} P<0.01$ versus W/W (OVA), ${ }^{N} P<0.01$ versus W/W (Saline). 


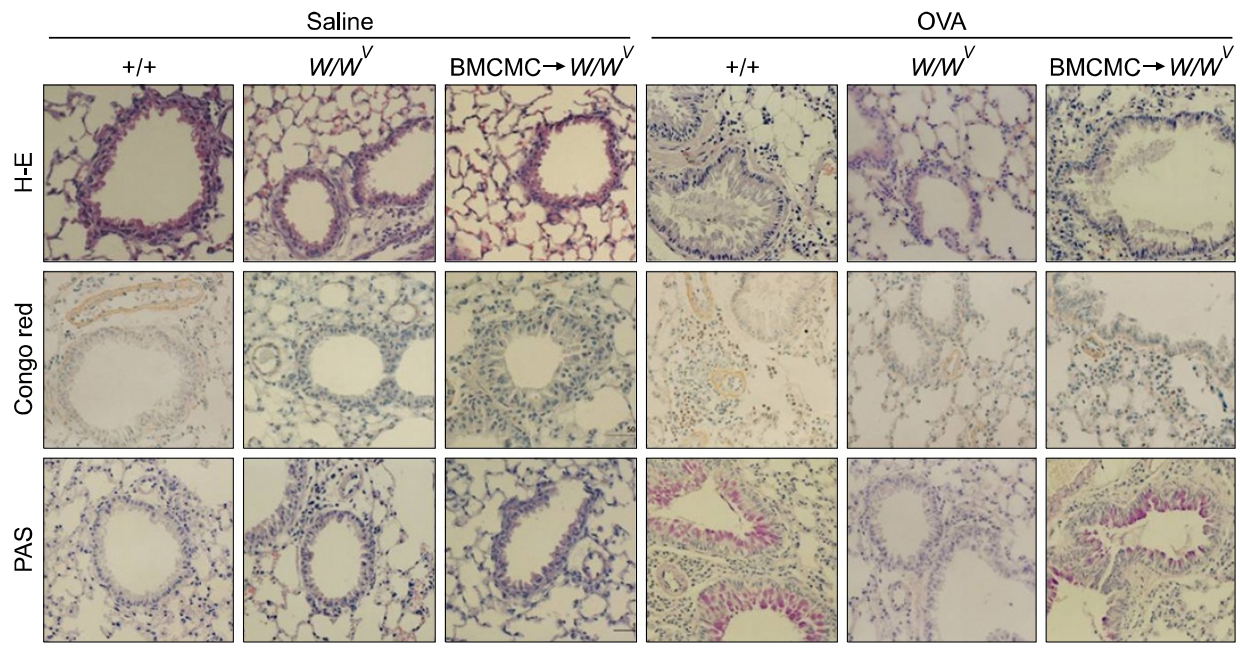

Figure 3. Histopathologic changes in lung tissues after saline sensitization and challenge (Saline) or ovalbumin sensitization and challenge (OVA) in $+/+, W / W^{N}$, and BMCMC $\rightarrow W / W^{N}$ mice. Lung tissues were stained with hematoxylin and eosin (H-E) for inflammatory cells, congo red for eosinophils, and periodic acid-Schiff (PAS) for goblet cells and mucus.

$\rightarrow W / W^{v}$ mice were restored to levels found in OVA-sensitized and -challenged +/+ mice.

\section{Cellular changes in mice after OVA challenge}

Numbers of macrophages, lymphocytes, neutrophils, eosinophils, and total cells in BAL fluids showed a significant increase at $48 \mathrm{~h}$ after OVA sensitization and challenge, compared with those after saline sensitization and challenge in $+/+$ mice (Figure 2). However, the numbers of macrophages, lymphocytes, neutrophils, eosinophils, and total cells in BAL fluids of OVA-sensitized and -challenged $W / W^{V}$ mice were significantly lower than in OVA-sensitized and -challenged $+/+$ mice. On the other hand, numbers of macrophages, lymphocytes, neutrophils, eosinophils, and total cells in $\mathrm{BAL}$ fluids of $\mathrm{BMCMC} \rightarrow W / W^{v}$ mice after OVA sensitization and challenge were significantly higher than in OVA-sensitized and -challenged $W / W^{v}$ mice.

\section{Histopathologic changes of lung challenged by OVA}

Histopathologic analyses revealed typical pathologic features of asthma in OVA-sensitized and -challenged mice (Figure 3). Lung tissues in mice given OVA were characterized by gross alterations in the structural integrity of the airway walls and parenchyma, epithelial cell shedding, microvascular leakage and extensive mucosal edema, increased tissue cellularity, and particulate exudated in airway lumina and alveolar septa. Numerous inflammatory cells, such as eosinophils, showed infiltration around bronchioles, mucus had accumulated in the lumen of bronchioles, and hyperplasia of goblet cells was observed at the epithelium of large airways after OVA sensitization and challenge, compared with those after saline sensitization and challenge in $+/+$ mice. However, infiltration of eosinophils around bronchioles, accumulation of mucus in the lumen of bronchioles, and hyperplasia of goblet cells at the epithelium of large airways of OVA sensitized and challenged $W W^{v}$ mice were significantly lower than in OVAsensitized and -challenged $+/+$ mice. On the other hand, infiltration of eosinophils around bronchioles, accumulation of mucus in the lumen of bronchioles, and hyperplasia of goblet cells at the epithelium of large airways of $\mathrm{BMCMC} \rightarrow W / W^{v}$ mice after OVA sensitization and challenge were significantly higher than in OVA-sensitized and -challenged $W / W^{v}$ mice.

\section{Levels of Th2 cytokines of BAL fluids in mice after OVA challenge}

Enzyme immunoassays showed that levels of TNF- $\alpha, I L-4$, and IL-5 protein in BAL fluids were significantly increased after OVA sensitization and challenge, compared with saline-sensitized and -challenged $+/+, W / W^{v}$, and $\mathrm{BMCMC} \rightarrow W / W^{v}$ mice (Figure 4). Levels of TNF- $\alpha$, IL-4, and IL-5 protein in BAL fluids in OVA-sensitized and -challenged $W / W^{v}$ mice were similar to levels found in saline-sensitized and -challenged $W / W^{V}$ mice. However, levels of TNF- $\alpha$, IL-4, and IL-5 protein in BAL fluids in OVA-sensitized and -challenged BMCMC 


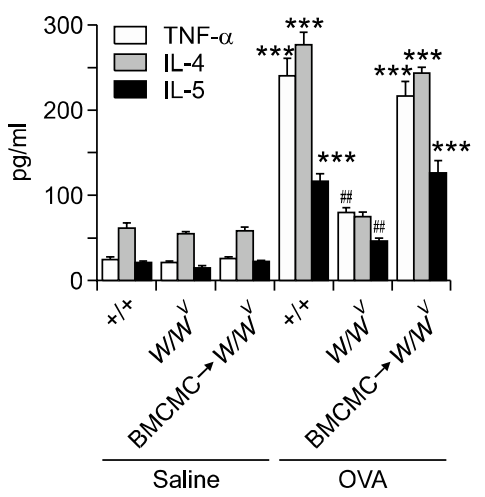

Figure 4. Enzymatic immunoassay for TNF- $\alpha$, IL-4, and IL-5 proteins in bronchoalveolar lavage fluid after saline sensitization and challenge (Saline) or ovalbumin sensitization and challenge (OVA) in $+/+, W / W^{N}$, and $\mathrm{BMCMC} \rightarrow W / W^{N}$ mice. ${ }^{* * *} P<0.001$ versus $W / W^{N}(\mathrm{OVA}),{ }^{\#} P<$ 0.01 versus $W / W^{N}$ (Saline).

$\rightarrow W / W^{V}$ mice had almost reached the levels found in OVA-sensitized and -challenged $+/+$ mice.

\section{ICAM-1 \& VCAM-1 expression in OVA challenged mice}

Two common and distinct pathways mediating mononuclear cell adhesion to pulmonary endothelium were investigated. Expression of endothelial ICAM-1 was observed in sections of blood vessels from lung of OVA-sensitized and -challenged $+/+$ mice and $\mathrm{BMCMC} \rightarrow W / W^{\nu}$ mice, whereas in saline-sensitized and -challenged +/+, $W / W^{\nu}$, and $\mathrm{BMCMC} \rightarrow W / W^{\nu}$ mice, ICAM-1 was hardly detected in endothelial cells of blood vessels. Also, ICAM-1 was hardly detected in endothelial cells of blood vessels of OVA-sensitized and -challenged $W / W^{\nu}$ mice (Figure $5 \mathrm{~A}$ ). In the contrast with ICAM-1, VCAM-1 expression was significantly heightened in vascular endothelium in blood vessels of OVA-sensitized and -challenged $+/+$ mice and $\mathrm{BMCMC} \rightarrow W / W^{\nu}$ mice and was most pronounced in vessels with perivascular inflammatory foci. Interestingly, VCAM-1 was also expressed on smooth muscle cells of blood vessels and bronchioles (Figure 5B). Whereas in saline-sensitized and -challenged $+/+, W / W^{\nu}$, and $\mathrm{BMCMC} \rightarrow W / W^{v}$ mice, VCAM-1 was hardly detected in endothelial cells of blood vessels. Also, VCAM-1 was hardly detected in endothelial cells of blood vessels of OVA-sensitized and -challenged $W / W^{v}$ mice. In BMCMC $\rightarrow W / W^{v}$ mice, expression of ICAM-1 and VCAM-1 in endothelial cells of blood vessels was restored to the levels found in OVAsensitized and -challenged +/+ mice (Figure 5B). Consistent with these results, western blot analysis revealed that levels of ICAM-1 and VCAM-1 pro- teins in lung tissue increased significantly with OVA challenge, compared with those after saline challenge in $+/+$ mice (Figure $5 \mathrm{C}$ ). However, levels of ICAM-1 and VCAM-1proteins in lung tissue of OVA-sensitized and -challenged $W / W^{V}$ mice were significantly lower than in lung tissue of OVAsensitized and -challenged $+/+$ mice. On the other hand, in $\mathrm{BMCMC} \rightarrow W / W^{V}$ mice, levels of ICAM-1 and VCAM-1 protein were restored to the level found in OVA-sensitized and -challenged $+/+$ mice (Figure 5C).

\section{Effects of TNF- $\alpha$ on ICAM-1 \& VCAM-1 protein levels in $W / W^{N}$ mice}

To investigate the effect of TNF- $\alpha$ on expression of ICAM-1 and VCAM-1 proteins, we assessed the
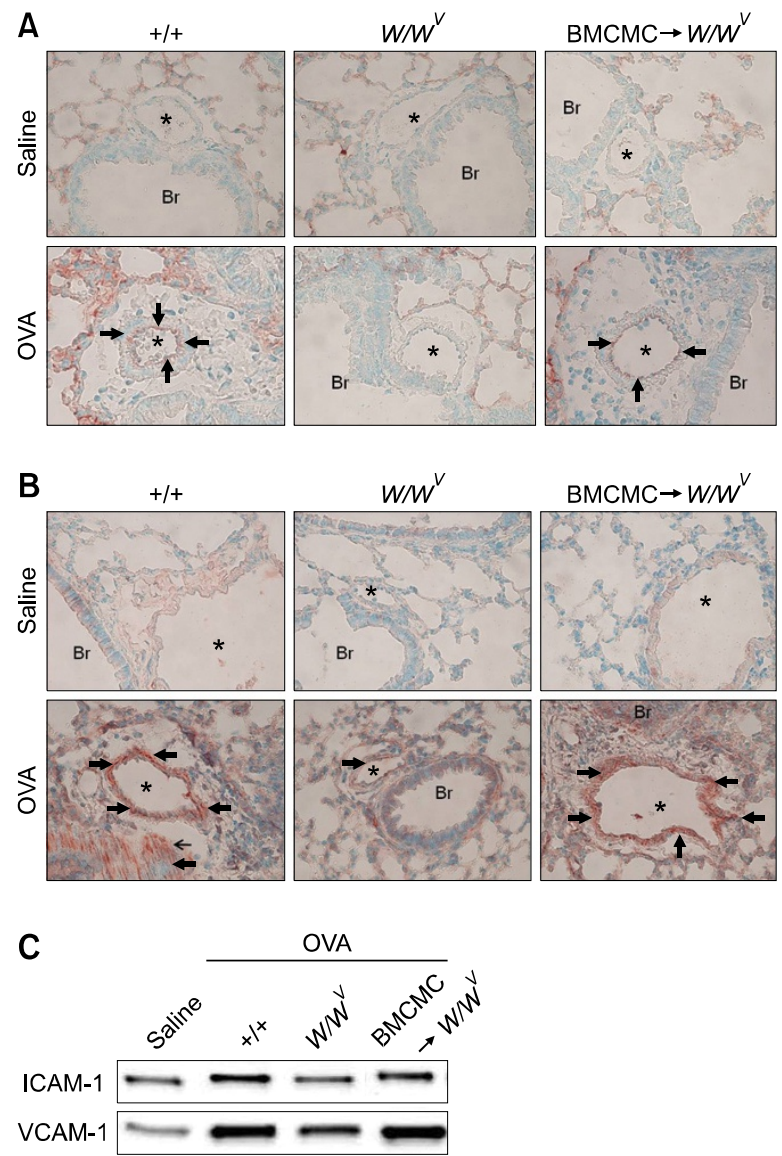

Figure 5. ICAM-1 and VCAM-1 proteins in lung tissues after saline sensitization and challenge (Saline) or ovalbumin sensitization and challenge $(\mathrm{OVA})$ in $+/+, W W^{N}$, and $\mathrm{BMCMC} \rightarrow W / W^{N}$ mice. Immunohistochemistry of (A) ICAM-1 (arrows) and (B) VCAM-1 (arrows) in endothelial cells and smooth muscle cells of blood vessels (*) of the lungs after saline sensitization and challenge or ovalbumin sensitization and challenge in $+/+$, $W / W^{N}$ and $B M C M C \rightarrow W / W^{N}$ mice. $B r$ indicates bronchioles. Arrow heads show immunoreactive ICAM-1 and VCAM-1. (C) Western blotting of ICAM-1and VCAM-1proteins in lung tissue. 
immunohistochemical analysis and western blot analysis of ICAM-1 and VCAM-1 proteins of lung tissue after intra tracheal injection of TNF- $\alpha$ in $W / W^{v}$ mice. Immunohistochemical analysis revealed that expressions of ICAM-1 and VCAM-1 were observed of in sections of blood vessels from lung of TNF- $\alpha$ intra tracheal injected $W / W^{v}$ mice. Localization of ICAM-1 and VCAM-1 expressions in endothelial layers around inflammation area and immunoreactive expression of VCAM-1 was more intensive than ICAM-1 (Figure 6A). Western blot analysis revealed that levels of ICAM-1 and VCAM-1 proteins were increased in a dose-dependent manner from lung of $W / W^{V}$ mice (Figure $6 \mathrm{~B}$ ).

\section{Discussion}

In this study, we investigated the possible roles of mast cells with regard to certain parameters of allergic inflammation in a Th2 cytokine-dependent asthma model using genetically mast cell-deficient $W / W^{v}$ mice, congenic normal $+/+$, and BMCMC $\rightarrow W / W^{V}$ mouse models. Our results suggest that mast cells play a key role in a Th2 cytokinedependent asthma model through production of VCAM- 1 and ICAM- 1 by release of TNF- $\alpha$.

Asthma is perceived as a Th2 disease with a particular profile of cytokine release, which is thought to include IL-4 and IL-5 (Woodruff et al., 2009). Increasing evidence indicates that other cytokines, which in mice are classically considered to belong to Th1-type profiles, are also associated with the inflammatory response that characterizes human asthma (Antunez et al., 2006). In the last decade it has become evident that cytokines play a pivotal role in the pathogenesis of asthma ( $\mathrm{Zi}$ mmermann et al., 2003; Antunez et al., 2006). The role of antigen-induced TNF- $\alpha$ release and antigen stimulation of other cytokines is an important area of study. In addition to TNF- $\alpha$, cytokines including IL-1 $\beta$, IL-2, IL-3, IL-4, IL-5, granulocyte-macrophage-colony stimulating factor, and interferon- $\gamma$ have also been implicated in development of the asthmatic inflammatory response (Thomas, 2001; Zimmermann et al., 2003; Antunez et al., 2006).

In this study, TNF- $\alpha$ showed an increase in BAL fluid after the second OVA challenge in $+/+$ mice, whereas OVA-sensitized and -challenged $W / W^{v}$ mice showed a significant decrease in TNF- $\alpha$ levels (up to $75 \%$ ) in their BAL fluid. In addition, TNF- $\alpha$ levels were significantly restored in BAL fluid in OVA-sensitized and -challenged BMCMC $\rightarrow W / W^{v}$ mice. Also, other cytokines, IL-4 and IL-5, showed a pattern similar to that of TNF- $\alpha$. Mast cell activation by specific antigen induces the cells to
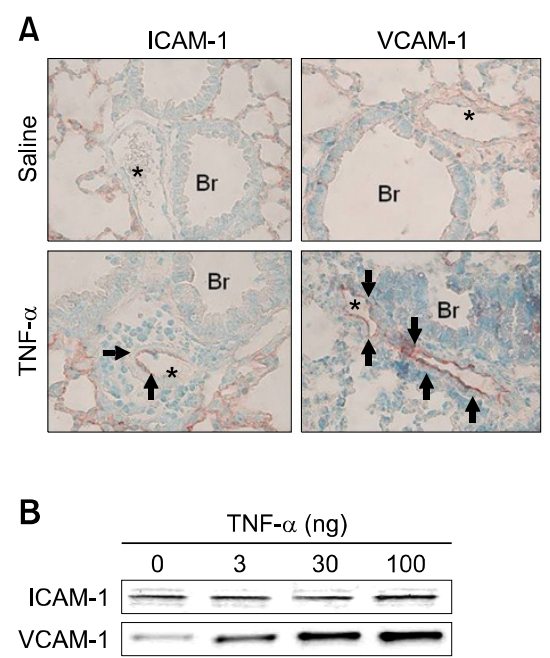

Figure 6. Effects of TNF- $\alpha$ on ICAM-1 \& VCAM-1 protein levels in W/W mice. (A) Immunohistochemistry of ICAM-1 and VCAM-1 (arrows). * indicates blood vessels of the lungs; $\mathrm{Br}$, bronchioles. (B) Western blotting of ICAM-1 and VCAM-1 $24 \mathrm{~h}$ after intra-tracheal injection of TNF- $\alpha$ in $W W^{N}$ mouse.

release spasmogenic, vasoactive and proinflammatory mediators, which in turn can enhance airway smooth muscle contraction, vascular permeability, and inflammatory cell ( $T$ cells, dendritic cell, neutrophils, eosinophils, and monocytes) recruitment at sites of antigen challenge (Biedermann et al., 2000; Bradding et al., 2006; Suto et al., 2006; Kneilling et al., 2009). Chemokines and cytokines are also released and induce neutrophil influx upon injection of cytokines, such as TNF- $\alpha$ (Ryan et al., 2007; Galli et al., 2008; Kawakami et al., 2009). On the other hand, locally released chemokines may activate resident cells to release cytokines. According to these reports, it is suggested that recruitment of inflammatory cells, activation and cytokine release from resident cells are consequent on mast cell activation by OVA. However, the asthma model used in this manuscript is mast cell-dependent, so there did not show the changes accompanied by a mast celldependent exacerbation of airway inflammation to OVA, and then did not induce cascade reactions such as, migration, local accumulation, and activation of proinflammatory cells and residential cell populations in the airway. So it is suggested that absence of mast cell result in a significant decrease in TNF- $\alpha, \mathrm{IL}-4$, and IL-5 levels. Our study provides evidence to show that cytokines play a pivotal role in the pathogenesis of asthma ( $\mathrm{Zi}-$ mmermann et al., 2003; Antunez et al., 2006).

Evidence for expression of ICAM-1 and VCAM-1 in smooth muscle cells in inflammatory diseases, such as arthrosclerosis and asthma, is mounting. 
The exact roles of expression of such adhesion molecules are not fully understood; however, it has been suggested that expression facilitates transmigration and accumulation of leukocytes at inflammatory sites. Increasing evidence has demonstrated the importance of cytokine-inducible leukocyte-endothelial adhesion molecules in recruitment and migration of leukocytes to sites of inflammation (Montefort and Holgate, 1991; Ando et al., 2001; Chihara, 2005). Expression of both ICAM-1 and VCAM-1 by smooth muscle cells in response to several inflammatory factors within airways has been reported in asthma animal models and allergic asthmatic patients (Gosset et al., 1995; Lee et al., 2003). In our study, we found that expression of ICAM-1 and VCAM-1 in lung tissue was significantly increased in OVA-sensitized and -challenged $+/+$ mice, compared with levels in OVA-sensitized and -challenged $W / W^{v}$ mice and saline-sensitized and -challenged +/+ mice. However, expression levels of ICAM-1 and VCAM-1 in lung tissue of OVA-sensitized and -challenged $\mathrm{BMCMC} \rightarrow W / W^{v}$ mice increased to levels similar to those found in OVA-sensitized and -challenged $+/+$ mice and was most pronounced in vessels with perivascular inflammatory foci. Interestingly, VCAM-1 was also expressed on smooth muscle cells of blood vessels and bronchioles. Therefore, our data suggest that ICAM-1 and VCAM-1 may play an important role in Th2 cytokine-dependent asthma and that mast cells may have an important action in induction and expression of ICAM-1 and VCAM-1 in Th2 cytokine-dependent asthma.

TNF- $\alpha$, a cytokine that plays a role in many inflammatory diseases, is produced mainly by macrophages; however, monocytes, dendritic cells, $B$ cells, mast cells, and eosinophils are also known to be crucial in the pathogenesis of asthma. Also, TNF- $\alpha$ is a chemotactic cytokine for granulocytes (Sabatini et al., 2002; Chai et al., 2005; Yoshifuku et al., 2007), probably by up-regulation of cellular adhesion molecules, such as E-selectin, ICAM-1 and VCAM-1, thus facilitating migration of eosinophils and neutrophils. During this process, they also become primed for mediator secretion. UItimately, this will lead to chronic inflammation and irreversible airway remodeling, a key feature in bronchial asthma. The role of TNF- $\alpha$ in allergic inflammation is still controversial. TNF- $\alpha$ induction of adhesion molecules in pulmonary endothelium is important for eosinophil recruitment (Wong et al., 2006); however, it has also been implicated in negative modulation of eosinophil level, IL-5 production, and, consequently, airway responsiveness (Kanehiro et al., 2001). In this study, we revealed the direct effect of TNF- $\alpha$ on up-regulation of ICAM-1 and VCAM-1 after intra tracheal injection of TNF- $\alpha$ in $W / W^{V}$ mice. Up-regulation of ICAM-1 and VCAM-1 increased in a dose manner of TNF- $\alpha$ in $W / W^{v}$ mice. This data showed that TNF- $\alpha$ induced adhesion molecules in the pulmonary endothelium of asthma.

TNF- $\alpha$ is stored in granules and is known to be released during allergic responses from both mast cells and macrophages via IgE-dependent mechanisms (Thomas, 2001; Zimmermann et al., 2003; Antunez et al., 2006). This finding indicated that this cytokine will be coreleased with more extensively characterized preformed mast cell granule mediators, such as histamine, chymase, and tryptase. Mast cell cytokines are predominantly Th2 cytokines, which are critical to development and maintenance of the asthmatic IgE-driven condition, and TNF- $\alpha$ has been shown to induce airway hyperreactivity. In our study, we demonstrated that expression of ICAM-1, VCAM-1, and protein in lung tissues was significantly increased after OVA sensitization and challenge of $+/+$ mice, compared with levels of saline sensitization and challenge in $+/+$ mice or OVA sensitization and challenge in $W / W^{V}$ mice. However, expression of ICAM-1, VCAM-1, and protein in lung tissue of OVA-sensitized and -challenged $\mathrm{BMCMC} \rightarrow W / W^{v}$ mice increased to levels similar to those found in OVA-sensitized and -challenged $+/+$ mice. Therefore, our results suggest that mast cell cytokines, particularly TNF- $\alpha$, are critical to development and maintenance of the asthma model through production of ICAM-1 and VCAM-1.

\section{Methods}

\section{Mice}

Genetically male mast cell-deficient mice $W / W^{N}$ mice and their congenic littermates $+/+$ mice, 5 weeks of age, were obtained from Japan SLC, Inc. $W / W^{N}$ mice ordinarily contain less than $1.0 \%$ of the number of dermal mast cells present in the skin of congenic normal $+/+$ mice and have no detectable mature mast cells in the respiratory system or other anatomic sites (Wolters et al., 2005; Galli and Tsai, 2008).

\section{Mast cell reconstitution}

Selective reconstitution of mast cells in mast cell deficient $W / W^{N}$ mice was carried out according to the method described by Williams and Galli (2000), with slight modifications. Suspended bone marrow cells from $+/+$ mice were cultured in WEHI-3 conditioned medium containing IL-3 for 4-5 weeks, at which time the cell populations were composed of $>95 \%$ immature mast cells, as assessed by 
staining with toluidine blue and Kimura staining. Five million bone marrow-derived cultured mast cells (BMCMCs) were infused via the tail vein into each $W / W^{N}$ mouse, and the recipients (BMCMC $\rightarrow W / W^{N}$ mice) were studied at least 16 wks later. There were $0.508 \pm 0.09$ mast cells $/ \mathrm{mm}^{2}$ in lung tissue of $\mathrm{BMCMC} \rightarrow W / W^{N}$ mice (vs none or $0.38 \pm$ 0.035 mast cells $/ \mathrm{mm}^{2}$ in lung tissue of $W / W^{\nu}$ or $+/+$ mice, respectively).

\section{Immunization and challenge with OVA}

$\mathrm{BMCMC} \rightarrow W / W^{\nu}$ and age-matched $+/+, W / W^{\nu}$ mice were used in these experiments. Mice were sensitized and challenged with ovalbumin (OVA) (Sigma-Aldrich Co., St. Louis, MO), as described previously, with some modifications (Williams and Galli, 2000; Kim et al., 2007) (Supplemental Data Figure S1). In brief, mice were immunized by intraperitoneal injection of $20 \mu \mathrm{g}$ OVA and saline in a total volume of $200 \mu$ on days 0 and 14 . Twenty eight days after the beginning of the sensitization period, these mice received their first intranasal challenge of $30 \mu \mathrm{l}$ of saline containing $200 \mu \mathrm{g}$ of OVA. After 7 days, these mice were challenged for $30 \mathrm{~min}$ with an aerosol of $5 \%$ $(w t / v o l)$ OVA in saline using ultrasonic nebulization (NE-U12; Omron Crop., Tokoyo, Japan).

\section{Measurement of airway reactivity}

Twenty four hours after the final OVA challenge, airway reactivity to aerosolized methacholine was measured using a whole body plethysmograph, as described by Ohtomo et al. (2009). In brief, unrestrained conscious mice were placed in whole body plethysmographic chambers, and, after $5 \mathrm{~min}$ of stabilization, dose response curves to aerosolized methacholine were generated. Increasing concentrations of methacholine were aerosolized for $3 \mathrm{~min}$ each, data for lung resistance and dynamic compliance were continuously monitored by using OCP 3000CPS-A software (allmedicus) and mean airway bronchoconstriction readings, as assessed by enhanced respiratory pause $(\mathrm{PenH})$, were obtained over $10 \mathrm{~min}$ periods. PenH can be conceptualized as the phase shift of the thoracic flow and nasal flow curves; increased phase shift correlates with increased respiratory system resistance. PenH is calculated by the formula $\mathrm{PenH}=(\mathrm{Te} / \mathrm{RT}-1) \times \mathrm{PEF} / \mathrm{PIF}$, where Te is expiratory time, RT is relaxation time, PEF is peak expiratory flow, and PIF is peak inspiratory flow.

\section{Collection and analysis of bronchoalveolar lavage (BAL) fluid}

Forty eight hours after the final OVA challenge, BAL fluid was collected by cannulating the upper part of the trachea and lavaging three times with $1 \mathrm{ml}$ saline. The lavage fluid collected was centrifugated at $400 \times g$ for $5 \mathrm{~min}$ at $4^{\circ} \mathrm{C}$, and the cells were separated from the fluid. The fluid was then centrifugated at $1,000 \times g$ for $2 \mathrm{~min}$ at $4^{\circ} \mathrm{C}$ for removal of cellular debris and then stored at $-20^{\circ} \mathrm{C}$ until it was evaluated. Cells were resuspended in PBS containing $1 \%$ FBS, and the total number of viable cells was determined by trypan blue exclusion using a hemocytometer.
Differential cell counts were determined with cytospin (Centrifuge 5403; Eppendorf, Hamburg, Germany) preparation, followed by Diff Quik staining (Sysmex Co., Kobe, Japan).

\section{Histopathology and immunohistochemistry}

Histopathologic analysis of lung and immunohistochemical examination of ICAM-1 and VCAM-1 were performed as previously described (Vrugt et al., 2000). Animals were sacrificed with an overdose of ether at $48 \mathrm{~h}$ after the last OVA exposure and histologic specimens were collected. Using routine histologic procedures for light microscopic evaluation, tissue specimens were taken from the mid zone of the left lung of the mice, and fixed in $10 \%$ formalin and embedded in paraffin. Serial sections measuring $5 \mu \mathrm{m}$ in thickness were then cut, and stained with hematoxylin and eosin for inflammatory cells, congo red for eosinophils, and periodic acid-Schiff for goblet cells and mucus. The others were stained with anti-ICAM-1 and anti-VCAM-1 antibodies.

\section{Measurements of Th2 cytokines in BAL fluids}

BAL was performed and BAL fluid was collected for analysis of Th2 cytokines in BAL fluid, smears of BAL cells were prepared by cytospin (Centrifuge 5403; Eppendorf, Hamburg, Germany). Levels of Th2 cytokines in supernatants of BAL fluids were quantified by mouse IL-4, IL-5, and TNF- $\alpha$ BD ELISA sets (BD PharMingen).

\section{Western blot analysis}

Mice lungs were minced and sonicated in Pro-Prep buffer (50 mg/ml) containing $50 \mathrm{mM}$ Tris-Cl, $\mathrm{pH}$ 7.5, $1 \mathrm{mM}$ EDTA, $150 \mathrm{mM} \mathrm{NaCl}$, and protease inhibitors using a tip-type sonicator (Branson sonifier, Branson ultrasonics, CT, 20\% amplitude, $3 \mathrm{~min}$ ), followed by centrifugation for $20 \mathrm{~min}$ at $1,000 \times g$. Protein expression levels in cell lysates were analyzed by Western blot analysis, as previously described (Vrugt et al., 2000; Lee et al., 2003). Equal amounts of cell lysate were separated on an SDS polyacrylamide gel under reducing conditions, and were then transferred onto Protran nitrocellulose membranes (Schleicher \& Schuell, Keene, $\mathrm{NH}$ ). Membranes were blocked for $2 \mathrm{~h}$ at room temperature in $3 \%$ skim milk in TBS, followed by overnight incubation with anti-TNF- $\alpha$ (BD PharMingen), anti-ICAM-1 (BD PharMingen), and anti-VCAM-1 (Santa Cruz). Blots were washed for 15 min with three changes of transfer buffer- $0.05 \%$ Tween 20 solution, followed by $1 \mathrm{~h}$ incubation at room temperature with HRP-conjugated anti-mouse antibody or anti-rabbit IgG antibody. Blots were washed again for $15 \mathrm{~min}$ and finally developed in ECL Western detection reagents (Amersham-Pharmacia Biotech, Piscataway, NJ). Signal intensities of specific bands were analyzed quantitatively using a Fluor-STM Imager (BioRad, Muncher, Germay) and plotted as relative intensity.

\section{Statistical analyses}

Unless otherwise specified, differences in response bet- 
ween different subgroups were tested for statistical significance using an unpaired Student's $t$ test (two tailed). $P$ $<0.05$ was considered statistically significant. Unless otherwise specified, all data are presented as mean \pm SEM.

\section{Acknowlegements}

1. $\mathrm{OH}$ Chai was supported by a Korea Research Foundation Grant funded by the Korean Government (MOEHRD, Basic Research Promotion Fund; KRF-2007-331-E00009). 2. $\mathrm{CH}$ Song was supported by a Korea Research Foundation Grant funded by the Korean Government (KRF-2008313-E00014).

\section{Supplemental data}

Supplemental Data include a figure and can be found with this article online at http://e-emm.or.kr/article/article_files/ SP-43-1-05.pdf.

\section{References}

Ando M, Shima M, Adachi M, Tsunetoshi Y. The role of intercellular adhesion molecule-1 (ICAM-1), vascular cell adhesion molecule-1 (VCAM-1), and regulated on activation, normal T-cell expressed and secreted (RANTES) in the relationship between air pollution and asthma among children. Arch Environ Health 2001;56:227-33

Antunez C, Torres MJ, Mayorga C, Corzo JL, Jurado A, Santamaria-Babi LF, Vera A, Blanca M. Cytokine production, activation marker, and skin homing receptor in children with atopic dermatitis and bronchial asthma. Pediatr Allergy Immunol 2006;17:166-74

Biedermann T, Kneilling M, Mailhanner R, Maier K, Sander CA, Kollias G, Kunkel SL, Hultner L, Rocken M. Mast cells control neutrophil recruitment during $T$ cell-mediated delayed type hypersensitivity reactions through tumor necrosis factor and mascrophage inflammatory protein 2. J Exp Med 2000;192:1441-51

Bradding $\mathrm{P}$, Walls AF, Holgate ST. The role of the mast cell in the pathophysiology of asthma. J Allergy Clin Immunol 2006; 117:1277-84

Chai OH, Lee HK, Lee YC, Lee MS, Han EH, Kim HT, Song $\mathrm{CH}$. Roles of TNF-alpha and IgE in the late phase of contact hypersensitivity induced by trimellitic anhydride. Exp Mol Med 2005;37:408-17

Chihara J. [Immunoglobulin super family (ICAM, VCAM, NCAM)]. Nippon Rinsho 2005;63 Suppl 8:139-41

Cohn L, Elias JA, Chupp GL. Asthma: mechanisms of disease persistence and progression. Annu Rev Immunol 2004;22:789-815

Galli SJ, Tsai M. Mast cells: versatile regulators of inflammation, tissue remodeling, host defense and homeostasis. J Dermatol Sci 2008;49:7-19

Galli SJ, Grimbaldeston M, Tsai M. Immunomodulatory mast cells: negative, as well as positive, regulators of immunity.
Nat Rev Immunol 2008;8:478-86

Gosset P, Tillie-Leblond I, Janin A, Marquette $\mathrm{CH}$, Copin MC, Wallaert B, Tonnel AB. Expression of E-selectin, ICAM-1 and VCAM-1 on bronchial biopsies from allergic and non-allergic asthmatic patients. Int Arch Allergy Immunol 1995;106:6977

Kanehiro A, Lahn M, Makela MJ, Dakhama A, Fujita M, Joetham A, Mason RJ, Born W, Gelfand EW. Tumor necrosis factor-alpha negatively regulates airway hyperresponsiveness through gamma-delta T cells. Am J Respir Crit Care Med 2001;164:2229-38

Kawakami T, Ando T, Kimura M, Wilson BS, Kawakami Y. Mast cells in atopic dermatitis. Curr Opin Immunol 2009; 21:666-78

Kim YS, Ko HM, Kang NI, Song $\mathrm{CH}$, Zhang X, Chung WC, Kim JH, Choi IH, Park YM, Kim GY, Im SY, Lee HK. Mast cells play a key role in the development of late airway hyperresponsiveness through TNF-alpha in a murine model of asthma. Eur J Immunol 2007;37:1107-15

Kim YS, Choi SJ, Choi JP, Jeon SG, Oh SY, Lee BJ, Gho YS, Lee CG, Zhu Z, Elias JA, Kim YK. IL-12-STAT4-IFN- $\gamma$ axis is a key downstream pathway in the development of IL-13mediated asthma phenotypes in a Th2 type asthma model. Exp Mol Med 2010;42:533-46

Kneilling M, Maihammer R, Hultner L, Schonberger T, Fuchs K, Schaller M, Bukala D, Massberg S, Sander CA, Braumuller $\mathrm{H}$, Eichner M, Maier KL, Hallmann R, Pichler BJ, Haubner R, Gawaz M, Pfeffer K, Biedermann T, Rocken M. Direct crosstalk between mast cell-TNF and TNFR1-expressing endothelia mediates local tissue inflammation. Blood 2009;114:1696-706

Lee KS, Jin SM, Kim HJ, Lee YC. Matrix metalloproteinase inhibitor regulates inflammatory cell migration by reducing ICAM-1 and VCAM-1 expression in a murine model of toluene diisocyanate-induced asthma. J Allergy Clin Immunol 2003;111:1278-84

Matsuoka T, Hirata M, Tanaka H, Takahashi Y, Murata T, Kabashima K, Sugimoto Y, Kobayashi T, Ushikubi F, Aze Y, Eguchi N, Urade Y, Yoshida N, Kimura K, Mizoguchi A, Honda $\mathrm{Y}$, Nagai H, Narumiya S. Prostaglandin D2 as a mediator of allergic asthma. Science 2000;287:2013-7

Montefort S, Holgate ST. Adhesion molecules and their role in inflammation. Respir Med 1991;85:91-9

Ohtomo T, Kaminuma O, Kitamura N, Suko M, Kobayashi N, Mori A. Murine Th clones confer late asthmatic response upon antigen challenge. Int Arch Allergy Immunol 2009;149 Suppl 1:2-6

Ryan JJ, Kashyap M, Bailey D, Kennedy S, Speiran K, Brenzovich J, Barnstein B, Oskeritzian C, Gomez G. Mast cell homeostasis: a fundamental aspect of allergic disease. Crit Rev Immunol 2007;27:15-32

Sabatini F, Silvestri M, Sale R, Scarso L, Defilippi AC, Risso FM, Rossi GA. Fibroblast-eosinophil interaction: modulation of adhesion molecules expression and chemokine release by human fetal lung fibroblasts in response to IL-4 and TNF-alpha. Immunol Lett 2002;84:173-8 
Suto H, Nakae S, Kakurai M, Sedgwick JD, Tsai M, Galli SJ. Mast cell-associated TNF promotes dendritic cell migration. J Immunol 2006;176:4102-12

Thomas PS. Tumour necrosis factor-alpha: the role of this multifunctional cytokine in asthma. Immunol Cell Biol 2001; 79:132-40

Vrugt B, Wilson S, Bron A, Holgate ST, Djukanovic R, Aalbers $R$. Bronchial angiogenesis in severe glucocorticoid-dependent asthma. Eur Respir J 2000;15:1014-21

Williams CM, Galli SJ. Mast cells can amplify airway reactivity and features of chronic inflammation in an asthma model in mice. J Exp Med 2000;192:455-62

Wolters PJ, Mallen-St Clair J, Lewis CC, Villalta SA, Baluk $\mathrm{P}$, Erle DJ, Caughey GH. Tissue-selective mast cell reconstitution and differential lung gene expression in mast cell-deficient Kit(W-sh)/Kit(W-sh) sash mice. Clin Exp Allergy 2005;35:82-8
Wong CK, Wang CB, Li ML, Ip WK, Tian YP, Lam CW. Induction of adhesion molecules upon the interaction between eosinophils and bronchial epithelial cells: involvement of p38 MAPK and NF-kappaB. Int Immunopharmacol 2006;6:1859-71

Woodruff PG, Modrek B, Choy DF, Jia G, Abbas AR, Ellwanger A, Koth LL, Arron JR, Fahy JV. T-helper type 2-driven inflammation defines major subphenotypes of asthma. Am J Respir Crit Care Med 2009;180:388-95

Yoshifuku K, Matsune S, Ohori J, Sagara Y, Fukuiwa T, Kurono Y. IL-4 and TNF-alpha increased the secretion of eotaxin from cultured fibroblasts of nasal polyps with eosinophil infiltration. Rhinology 2007;45:235-41

Zimmermann N, Hershey GK, Foster PS, Rothenberg ME. Chemokines in asthma: cooperative interaction between chemokines and IL-13. J Allergy Clin Immunol 2003;111: $227-42$ 